\title{
Globular Clusters in NGC 5128: The IMACS survey $\dagger$
}

\author{
M. Gómez ${ }^{1}$, D. Geisler ${ }^{1}$, W. E. Harris ${ }^{2}$, G. L. H. Harris ${ }^{3}$, \\ and K. A. Woodley ${ }^{2}$
}

${ }^{1}$ Depto. de Física, Univ. de Concepción, Casilla 160-C, Concepción, Chile email: matias@astro-udec.cl

${ }^{2}$ Department of Physics and Astronomy, McMaster University, Hamilton ON L8S 4M1, Canada

${ }^{3}$ Department of Physics, University of Waterloo, Waterloo, Ontario, N2L 3G1, Canada

Abstract. To extend our recent study of structural parameters of Globular Clusters (GCs) in the giant elliptical NGC 5128 (Gómez et al. 2006), we have obtained new high resolution images with the Magellan $6.5 \mathrm{~m}$ at Las Campanas Observatory and the IMACS camera. Our images cover an area of $1.2 \times 1.2 \mathrm{sq}$. degrees and have a seeing between 0.4 and $0.7^{\prime \prime}$, thus making it possible to resolve a significant fraction of the GC population, which is now virtually entirely observed given the large spatial and magnitude range.

Convolution techniques allow us to find and to study the cluster candidates using the stellar PSF and analytical profiles for their light distribution. We therefore are able to derive structural parameters like size (effective and core radii), ellipticity, position angle and concentration. This is combined with the extensive database in the Washington photometric system by Harris et al. (2004) to select clusters according to shape, extendedness and Washington magnitudes and colours, which has proven very useful in discriminating GCs from foreground stars and background galaxies and for deriving metallicities.

Our first results for the central field $\left(15^{\prime} \times 15^{\prime}\right)$ indicate that the GCs populate a large range of $\left(M_{V}, r_{h}\right)$ parameter space, with typical sizes of Galactic GCs as well as much larger. Combined with our previous data, we find a continuum of clusters in this diagram, with no clear distinction between a variety of different 'types' often referred to, such as UCDs, Faint Fuzzies, etc.

In addition, a large fraction of GCs show a significant ellipticity whereas a paucity of round clusters is observed. We also confirm with a large $(\sim 250)$ sample that metal-rich clusters are smaller than metal-poor ones, which is in keeping with the general trend found in other galaxies. (e.g. Jordán et al. 2005)

Keywords. galaxies: star clusters, galaxies: individual (NGC 5128)

\section{References}

Gómez, M., Geisler, D., Harris, W. E., et al. 2006, A\&A 447, 877.

Harris, G. L. H., Geisler, D., Harris, W. E., et al. 2004, AJ 128, 712.

Jordán, A., Cote, P., Blakeslee, J. P., et al. 2005, ApJ 634, 1002.

$\dagger$ This paper includes data gathered with the 6.5 meter Magellan Telescopes located at Las Campanas Observatory, Chile. 